\title{
Protostellar holes: Spitzer Space Telescope observations of the protostellar binary IRAS 16293-2422
}

\author{
Jes K. Jørgensen ${ }^{1}$, Fred Lahuis ${ }^{2,3}$, Fredrik L. Schöier ${ }^{4}$, Ewine F. van Dishoeck ${ }^{2}$, Geoffrey A. \\ Blake $^{5}$, A. C. Adwin Boogert ${ }^{6}$, Cornelis P. Dullemond ${ }^{7}$, Neal J. Evans II ${ }^{8}$, Jacqueline E. \\ Kessler-Silacci ${ }^{8}$, and Klaus M. Pontoppidan ${ }^{2}$
}

\begin{abstract}
Mid-infrared (23-35 $\mu \mathrm{m})$ emission from the deeply embedded "Class 0" protostar IRAS 16293-2422 is detected with the Spitzer Space Telescope infrared spectrograph. A detailed radiative transfer model reproducing the full spectral energy distribution (SED) from $23 \mu \mathrm{m}$ to $1.3 \mathrm{~mm}$ requires a large inner cavity of radius $600 \mathrm{AU}$ in the envelope to avoid quenching the emission from the central sources. This is consistent with a previous suggestion based on high angular resolution millimeter interferometric data. An alternative interpretation using a 2D model of the envelope with an outflow cavity can reproduce the SED but not the interferometer visibilities. The cavity size is comparable to the centrifugal radius of the envelope and therefore appears to be a natural consequence of the rotation of the protostellar core, which has also caused the fragmentation leading to the central protostellar binary. With a large cavity such as required by the data, the average temperature at a given radius does not increase above $60-80 \mathrm{~K}$
\end{abstract}

\footnotetext{
${ }^{1}$ Harvard-Smithsonian Center for Astrophysics, 60 Garden Street, MS42, Cambridge, MA 02138 (jjorgensen@cfa.harvard.edu)

${ }^{2}$ Leiden Observatory, PO Box 9513, 2300 RA Leiden, The Netherlands (freddy@sron.rug.nl; ewine, pontoppi@strw.leidenuniv.nl)

${ }^{3}$ SRON National Institute for Space Research, PO Box 800, 9700 AV Groningen, The Netherlands

${ }^{4}$ Stockholm Observatory, AlbaNova, 10691 Stockholm, Sweden (fredrik@astro.su.se)

${ }^{5}$ Division of Geological and Planetary Sciences, Mail Code 150-21, California Institute of Technology, Pasadena, CA 91125 (gab@gps.caltech.edu)

${ }^{6}$ Division of Physics, Mathematics and Astronomy, Mail Code 150-24, California Institute of Technology, Pasadena, CA 91125 (acab@astro.caltech.edu)

${ }^{7}$ Max Planck Institut für Astronomie, Koenigstuhl 17, 69117 Heidelberg, Germany (dullemon@mpia.de)

${ }^{8}$ Department of Astronomy, University of Texas at Austin, 1 University Station, C1400, Austin, TX 78712 (nje, jes@as.utexas.edu)
} 
and although hot spots with higher temperatures may be present close to each protostar, these constitute a small fraction of the material in the inner envelope. The proposed cavity will also have consequences for the interpretation of molecular line data, especially of complex species probing high temperatures in the inner regions of the envelope.

Subject headings: star: formation — stars: individual(IRAS 16293-2422)

\section{Introduction}

In the earliest stages of their evolution, low-mass young stellar objects are thought to be deeply embedded in an envelope of cold gas and dust. The objects therefore emit large fractions of their emission at far-infrared through submillimeter wavelengths. André et al. (1993) introduced the term "Class 0" for such protostars with $L_{\text {submm }} / L_{\text {bol }}>0.5 \%$ where $L_{\text {submm }}$ is the luminosity originating longwards of $350 \mu \mathrm{m}$. The typical mid-infrared 10$20 \mu \mathrm{m}$ fluxes of these sources are predicted to be very weak, below $1 \mathrm{mJy}$. With the Spitzer Space Telescope's high sensitivity, it now becomes possible to search for these objects in the mid-infrared and test models for their structure. This paper presents Spitzer/IRS observations of the deeply embedded low-mass protostar IRAS 16293-2422. These observations, together with a detailed dust radiative transfer model (Schöier et al. 2002, 2004), constrain the properties of the innermost region of the protostellar envelope.

In recent years, a substantial step forward in studies of deeply embedded protostars is the quantitative characterization of the physical structure of their envelopes through observations with submillimeter cameras combined with dust radiative transfer models (e.g., Shirley et al. 2000, 2002; Motte \& André 2001; Jørgensen et al. 2002). The submillimeter observations are predominantly sensitive to the cold material in the outer envelope, which is why the radiative transfer models constrain the structure of the envelope, such as its density and temperature profiles, on larger scales. Thus, the mid-infrared data probing the inner warm region are highly complementary.

Of all the protostars studied in this manner, IRAS 16293-2422 has gained particular attention: it is a protobinary with a separation of $\sim 800 \mathrm{AU}$ (Mundy et al. 1992) and a total luminosity of $27 L_{\odot}$. With this luminosity, material in the inner $\sim 100 \mathrm{AU}$ of the envelope will have temperatures higher than $\sim 100 \mathrm{~K}$ due to the heating from the central protostar(s) (Schöier et al. 2002). Interpretations of, e.g., the chemistry in these regions rely on extrapolation of the larger scale envelope parameters to small scales. A way to test the extrapolation is through aperture-synthesis observations of the dust continuum emission compared to the 
predictions from the models. Schöier et al. (2004) analyzed $1 \mathrm{~mm}$ observations from the Owens Valley Radio Observatory Millimeter Array and suggested that the envelope around IRAS 16293-2422 in fact has an inner cavity with a size corresponding to the well resolved binary. The projected binary separation of 800 AU would suggest a minimum inner radius for the envelope of 400 AU because the binary would clear a cavity through dynamical interaction. Still, these data are mostly sensitive to the cold dust in the envelope. Independent constraints can be obtained at mid-infrared wavelengths where it is possible to study the warm dust close to the central protostar. IRAS 16293-2422 is also known to drive prominent outflows (Walker et al. 1988), and the associated cavities may further allow the mid-infrared radiation to escape.

\section{Observations}

IRAS 16293-2422A was observed by the "Cores to Disks" (c2d) legacy team (Evans et al. 2003) using the infrared spectrograph (IRS) on 2004 August 29 (AOR key 11826944). The source was observed in staring mode with the SH and LH modules $(9.9-19.6 \mu \mathrm{m}$ and $18.7-37.2 \mu \mathrm{m} ; R \sim 600)$ and SL1 and SL2 modules $(5.2-14.5 \mu \mathrm{m} ; R \sim 64-128)$. The observations were centered on the south eastern component in the protostellar binary at $\alpha_{2000}=16^{\mathrm{h}} 32^{\mathrm{m}} 22^{\mathrm{s}} .87, \delta_{2000}=-24^{\circ} 28^{\prime} 36^{\prime \prime} .1$ (Looney et al. 2000).

Data reduction started from the BCD images using S10.5.0 Spitzer archive data. The processing includes bad-pixel correction, extraction, defringing and order matching using the c2d analysis pipeline (Lahuis et al. in prep.). The source is not detected shortward of $23 \mu \mathrm{m}$ but rises steeply from 23-35 $\mu \mathrm{m}$ (Fig. 1). For these wavelengths an optimized source profile extraction was used to separate the source from the extended cloud emission. $\mathrm{H}_{2}$ and $\mathrm{PAH}$ emission is also detected: both are extended and not directly associated with the source.

\section{Dust radiative transfer model}

\subsection{Background}

The model for the outer envelope structure by Schöier et al. (2002) cannot reproduce the mid-infrared flux seen by Spitzer (Fig. 1) and we therefore reexamine these models. In short, such models calculate the dust radiative transfer through a spherical envelope illuminated by a central source supplying the total luminosity $L$ using the $1 \mathrm{D}$ radiative transfer code Dusty (Ivezić et al. 1999). The inputs into the models are the spectrum of the central source (approximated as a blackbody with a specific effective temperature, $T_{\text {eff }}$ ), the inner and outer 
radii of the envelope, $R_{\mathrm{i}}$ and $R_{\mathrm{e}}$, and the density distribution of the envelope. The density distribution is typically described by a power-law profile, $\rho=\rho_{0}\left(r / r_{0}\right)^{-p}$, leaving two free parameters: the slope $p$ and normalization, $\rho_{0}$. The latter can be specified through the total dust optical depth at a specified wavelength (e.g., $\tau_{100}$ for the optical depth at $\left.100 \mu \mathrm{m}\right)$. The spectrum of the central source and the inner radius of the envelope are not constrained by the submillimeter emission. As in Schöier et al. (2002) and Jørgensen et al. (2002) we adopt the opacities of coagulated dust grains with thin ice mantles at a density of $10^{6} \mathrm{~cm}^{-3}$ from Ossenkopf \& Henning (1994). For further discussions of the models we refer to the cited papers.

The detection of IRAS 16293-2422 in the mid-infrared suggests that the envelope surrounding the source is less optically thick at these wavelengths compared to the model of Schöier et al. (2002), which severely underestimates the emission at mid-infrared wavelengths (Fig. 1). Its dust optical depth toward the central illuminating source is $\approx 40$ at $25 \mu \mathrm{m}$. One option to reproduce the entire SED is to increase the inner radius of the envelope. This is consistent with the high angular resolution millimeter interferometric data by Schöier et al. (2004) who suggested the presence of an inner cavity in the envelope with a radius of $\approx 400 \mathrm{AU}$ resolved by the interferometer. With such a cavity, the column density of material along the line of sight is decreased, whereas the total mass of the envelope and thereby the submillimeter emission remains largely unaffected.

\subsection{Results}

To test the idea of a larger inner radius, a grid of models is calculated varying $R_{\mathrm{e}}$ and $\tau_{100}$ as in Schöier et al. - but now also letting $R_{\mathrm{i}}$ vary. The slope of the density distribution, $p$, is fixed to 1.9, which provides a good fit to the brightness distribution in single-dish observations on a few thousand AU scales and aperture synthesis observations on a few hundred AU scales (Schöier et al. 2002, 2004). To perform the fits and estimate the $\chi^{2}$ statistic, the mid-infrared spectrum is rebinned into fourteen $\Delta \lambda=1 \mu \mathrm{m}$ bins. The error in each measurement is taken to be the standard deviation of the flux measurements in each bin with an addition of $10 \%$ of the resulting flux, representing the uncertainty in the absolute flux calibration and relative calibration across the spectrum.

The $\chi^{2}$ confidence plots are shown in Fig. 2. As panels a)-c) illustrate, the mid-infrared SED provides good constraints on the size of the inner cavity whereas it is completely insensitive to the emission from the outermost regions of the envelope. Both the submillimeter and mid-infrared SEDs are well fitted by a model similar to that presented in Schöier et al. (2002) with the additional constraint from the IRS spectrum that $R_{\mathrm{i}}=600_{-150}^{+250}$ AU (corre- 
sponding to $\tau_{100}=0.5_{-0.10}^{+0.05}$ ) (Fig. 2c). The reduced $\chi^{2}$ for the best fit model is $\approx 2.5$ and $\lesssim 1$ for the fits to the mid-infrared and far-infrared/submillimeter parts of the SED, respectively. A cavity of 400 AU radius suggested by the model of Schöier et al. (2004) is not ruled out by the mid-infrared observations (Fig. 2). That model fits the submillimeter SED and millimeter interferometer data which resolve the envelope cavity. Still, the mid-infrared data place stronger constraints on the thickness of the envelope in that particular case compared to just considering the submillimeter SED.

One natural concern is whether the spectral shape of the central source influences the result. In particular, the central circumstellar disks could be bright at mid-infrared wavelengths compared to the stellar blackbodies. Still, if the envelope is highly optically thick at 23-35 $\mu \mathrm{m}$ the effect of changing the SED of the central source will be small. Fig. $2 \mathrm{~d}$ illustrates the extreme example where the total luminosity of the central object is coming from a $100 \mathrm{~K}$ blackbody. The change in spectrum affects the larger scale emission of the envelope somewhat in the optically thin (long wavelength) regime but the requirement that the envelope only is marginally optically thick at $23-35 \mu \mathrm{m}$ still constrains the minimum cavity size.

\subsection{Going to $2 \mathrm{D}$ ?}

Another question is whether the 1D models truly represent the structure of the envelopes at the level of detail suggested by these observations. An alternative explanation for the increased mid-infrared radiation is that the $1 \mathrm{D}$ models are not a good representation of the structure of the envelope, since they do not contain any outflow cavities. Whitney et al. (2003) showed that the mid-infrared SED strongly depends on the inclination angle if a large outflow cavity is present in the envelope. To illustrate the sensitivity to the inclination angle, a $2 \mathrm{D}$ radiative transfer model was calculated using a dust Monte Carlo code (Dullemond \& Turolla 2000; Dullemond \& Dominik 2004). In this model, a conical slice of the envelope is cut out mimicking an outflow cavity. The cavity is assumed to have an opening angle of $30^{\circ}$ and to be completely devoid of material. Otherwise the $2 \mathrm{D}$ structure is identical to the $1 \mathrm{D}$ model of Schöier et al. (2002), i.e., extending inwards to about 30 AU. Fig. 3 compares the $\mathrm{SED}$ for the 2D model with varying inclination angles. For lines-of-sight down or close to the cavity the mid-infrared flux is indeed increased and a good fit to the mid-infrared SED is obtained with an inclination angle of $17-20^{\circ}$.

Introducing the outflow cavity, however, does not alleviate the need for an inner cavity inferred from the high angular resolution millimeter data: Fig. 4 compares the predicted visibilities at $1.37 \mathrm{~mm}$ from the $1 \mathrm{D}$ envelope models with increasing cavity sizes and the 2D 
model presented above. The 1D spherical model with a small inner cavity and the similar model with an outflow cavity are virtually indistinguishable and both produce too much flux on intermediate baselines $(10-30 \mathrm{k} \lambda)$. In contrast the visibilities from the models of Schöier et al. (2004) and this paper drop quickly at these projected baselines, as required by comparison to the interferometer data.

The mid-infrared and high angular resolution millimeter data are complementary: the best constraints on the 1D cavity model are obtained from the mid-infrared data probing the warm dust but the existence of the inner cavity is demonstrated by the millimeter interferometer data (which also provides constraints on the circumstellar disks embedded in the envelope). It should be emphasized that the data do not exclude the presence of an outflow cavity, but that an inner cavity is still required even though an outflow cavity can reproduce the mid-infrared data. An even more complicated multi-dimensional structure can also not be excluded.

\section{Discussion}

The inner cavity in the IRAS 16293-2422 envelope inferred here is of comparable size to the separation of the binaries. Does this simply reflect the centrifugal radius due to rotation in the core before the collapse into the binary? Zhou (1995) examined large scale CS single-dish observations and found that two regimes of rotation applied to the IRAS 162932422 core indicative of differential rotation: the inner region of the envelope rotating with $\Omega=3 \times 10^{-13} \mathrm{~s}^{-1}$ and the outer region about factor 6 slower. A similar rotation rate in the inner envelope was found through single-dish observations by Narayanan et al. (1998). From comparison of the line observations to the Terebey et al. (1984) models of slowly rotating, infalling cores, Zhou found a centrifugal radius (where gravity is balanced by rotation) of $600 \mathrm{AU}$ (corresponding to a collapse timescale of $1 \times 10^{5}$ years in the Terebey et al. (1984) model for the sound speed derived by Zhou). This similarity to the inner radius derived from the mid-infrared observations is striking: the requirement of an inner envelope cavity

from the strength of the mid-infrared emission may simply reflect the centrifugal radius of the protostellar envelope.

Boss (1999) examined the properties of rotating collapsing cores. In these simulations cores with similar ratios between their rotation and gravitational energy such as IRAS 162932422 ( $\beta=0.03$; based on the estimates of the rotation rate by Zhou (1995) and the envelope structure in this paper) do indeed collapse and form a binary. It therefore seems that the conclusion about the inner cavity in the IRAS 16293-2422 envelope is a natural consequence of its rotation, which has also resulted in the protostellar binary. On the other hand, if the 
binary had formed by other means, the dynamical interaction between the binary components and the envelope would likely have resulted in clearing of the inner cavity.

The temperatures and densities in the outer envelope are very similar in both models with small and large inner cavities. The interpretation of the chemistry of molecular species probing these regions will therefore not be affected by the revised models in Schöier et al. (2004) and this paper. The interpretation of line emission coming from the inner "hot core" (e.g., Ceccarelli et al. 2000; Schöier et al. 2002) will clearly be affected, however. In the cavity model preferred here, the envelope temperature does not rise above 60-80 K. Locally, of course, the temperature will be higher since the binary separation is comparable to the cavity size. In particular, at the edge of the cavity close to each of the binary components the temperature should be higher than that obtained from the spherically symmetric calculations, whereas in orthogonal directions to the binary the temperature is expected to be lower. However, since each binary component can heat the envelope material above $100 \mathrm{~K}$ only within about $100 \mathrm{AU}$, it will be a small fraction of the envelope which has temperatures higher than $100 \mathrm{~K}$ typical of a "hot core". It therefore appears likely that the complex organic species have a different origin than passively heated material in the protostellar envelope. An alternative could be accretion shocks in each of the circumstellar disks or, as suggested by the high angular resolution submillimeter observations by Chandler et al. (2005), a result of the shock associated with the IRAS 16293-2422A outflow.

The research of JKJ was supported by NASA Origins Grant NAG5-13050. Support for this work, part of the Spitzer Legacy Science Program, was also provided by NASA through contract 1224608 issued by the Jet Propulsion Laboratory, California Institute of Technology, under NASA contract 1407. Astrochemistry research in Leiden is supported by a NWO Spinoza grant and a NOVA grant. FLS acknowledges financial support from the Swedish Research Council. 


\section{REFERENCES}

André, P., Ward-Thompson, D., \& Barsony, M. 1993, ApJ, 406, 122

Boss, A. P. 1999, ApJ, 520, 744

Ceccarelli, C., Castets, A., Caux, E., Hollenbach, D., Loinard, L., Molinari, S., \& Tielens, A. G. G. M. 2000, A\&A, 355, 1129

Chandler, C. J., Brogan, C. L., Shirley, Y. L., \& Loinard, L. 2004, ApJ, in press. (astro$\mathrm{ph} / 0506435)$

Dullemond, C. P., \& Dominik, C. 2004, A\&A, 417, 159

Dullemond, C. P., \& Turolla, R. 2000, A\&A, 360, 1187

Evans, N. J., et al. 2003, PASP, 115, 965

Ivezić, Ž., Nenkova, M., \& Elitzur, M. 1999, User Manual for DUSTY, University of Kentucky Internal Report

Jørgensen, J. K., Schöier, F. L., \& van Dishoeck, E. F. 2002, A\&A, 389, 908

Looney, L. W., Mundy, L. G., \& Welch, W. J. 2000, ApJ, 529, 477

Motte, F., \& André, P. 2001, A\&A, 365, 440

Mundy, L. G., Wootten, A., Wilking, B. A., Blake, G. A., \& Sargent, A. I. 1992, ApJ, 385, 306

Narayanan, G., Walker, C. K., \& Buckley, H. D. 1998, ApJ, 496, 292

Ossenkopf, V., \& Henning, T. 1994, A\&A, 291, 943

Schöier, F. L., Jørgensen, J. K., van Dishoeck, E. F., \& Blake, G. A. 2002, A\&A, 390, 1001

Schöier, F. L., Jørgensen, J. K., van Dishoeck, E. F., \& Blake, G. A. 2004, A\&A, 418, 185

Shirley, Y. L., Evans, N. J., \& Rawlings, J. M. C. 2002, ApJ, 575, 337

Shirley, Y. L., Evans, N. J., Rawlings, J. M. C., \& Gregersen, E. M. 2000, ApJS, 131, 249

Terebey, S., Shu, F. H., \& Cassen, P. 1984, ApJ, 286, 529

Walker, C. K., Lada, C. J., Young, E. T., \& Margulis, M. 1988, ApJ, 332, 335 
Whitney, B. A., Wood, K., Bjorkman, J. E., \& Wolff, M. J. 2003, ApJ, 591, 1049

Zhou, S. 1995, ApJ, 442, 685 

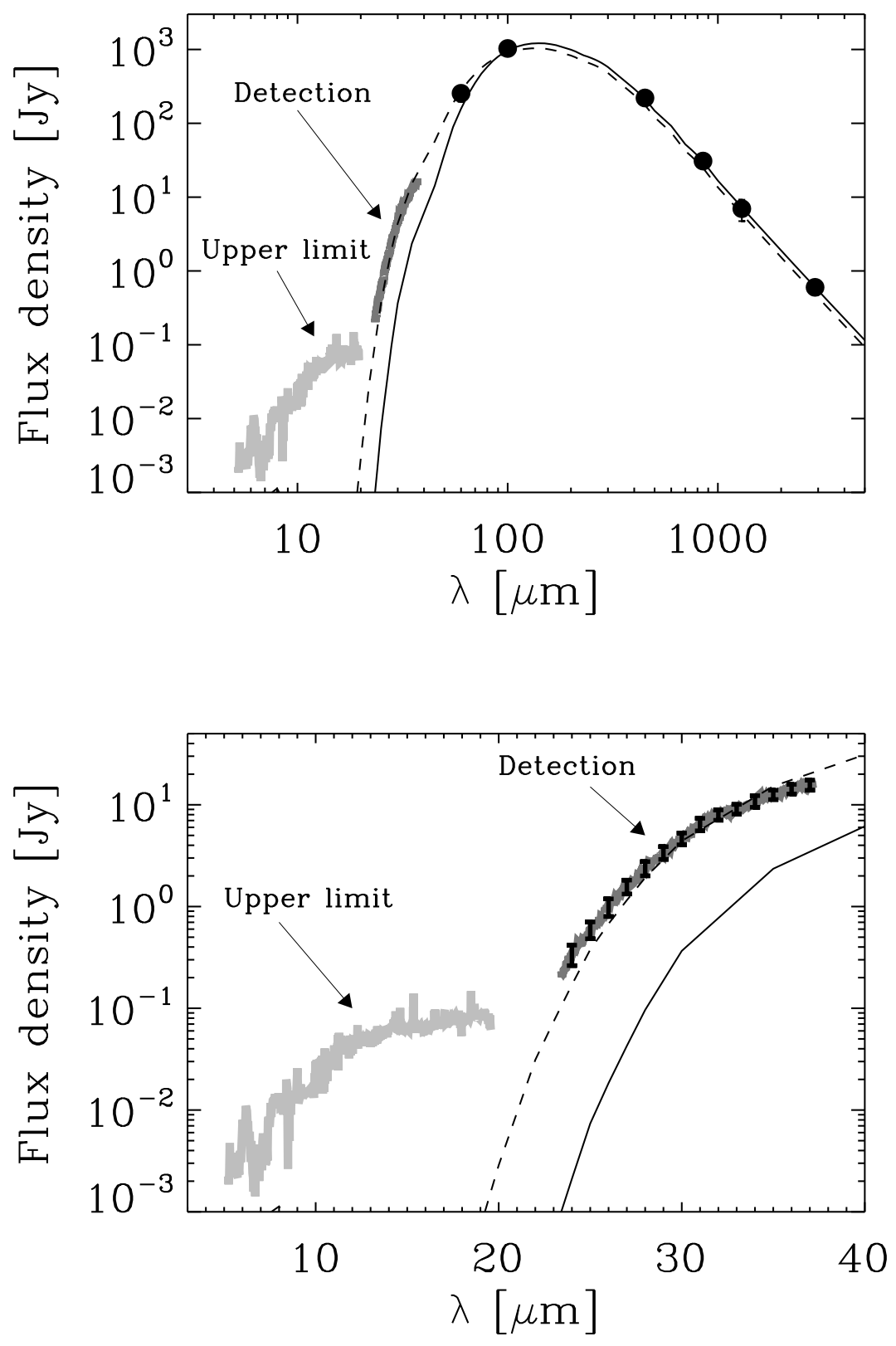

Fig. 1. - Spitzer/IRS observations of IRAS 16293-2422 and models for its SED. In both panels the dark grey line indicates the IRS detection and the light grey line the upper limit. The black solid line shows the model of Schöier et al. (2002), the dashed line the best fit model from this paper. In the upper panel the symbols indicate the far-infrared/submillimeter SED from Schöier et al.. In the lower (blow-up) panel the symbols indicate the bins of the IRS spectrum used in the fits. 

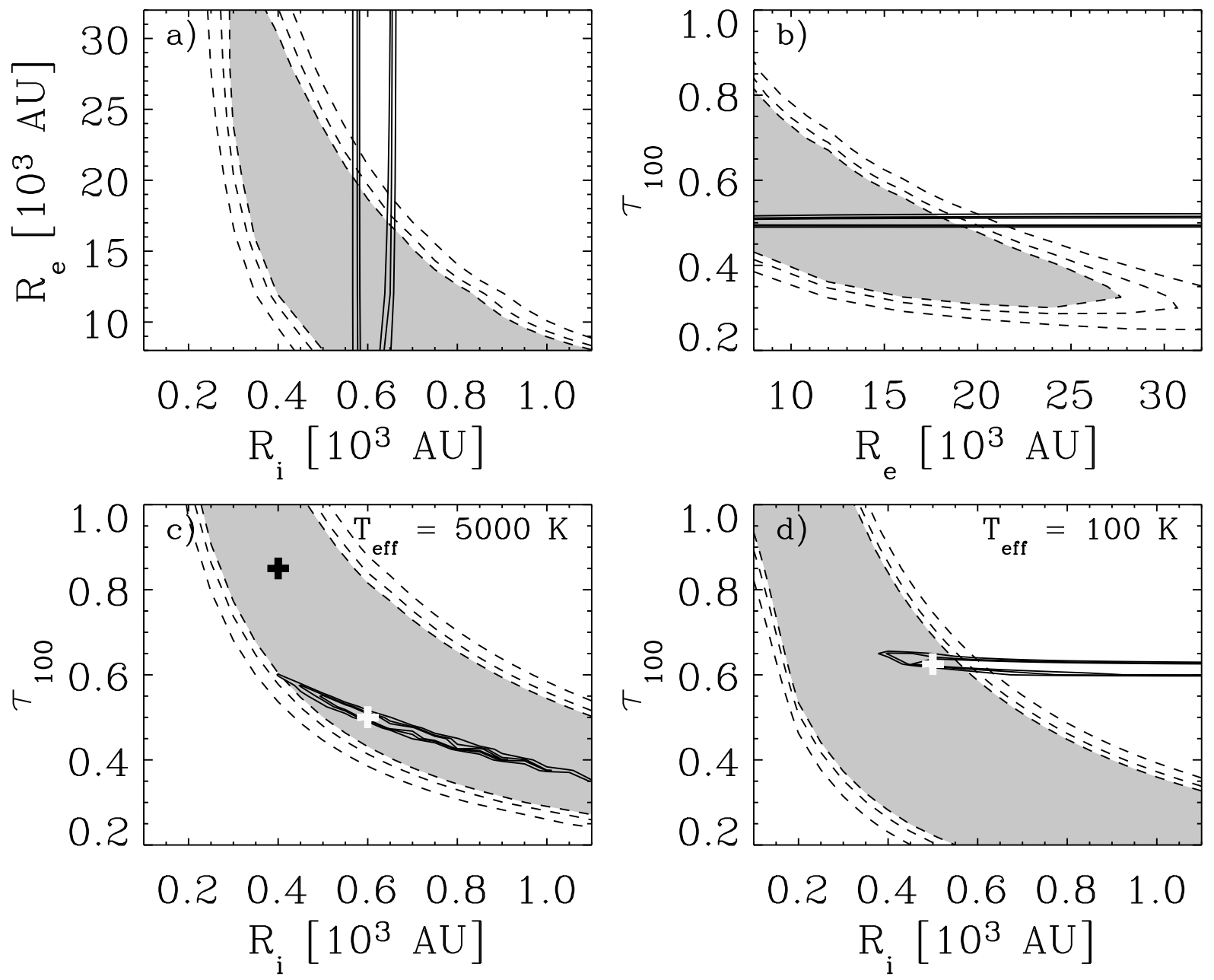

Fig. 2.- $\chi^{2}$-confidence plots from fits to the SED with the envelope model. In each panel the contours indicate the $90 \%, 95 \%$ and $99 \%$ confidence limits. The dashed line contours indicate the fits to the far-infrared/submillimeter SED (Schöier et al. 2002) whereas the solid line contours indicate the fits to the Spitzer/IRS mid-infrared SED. The grey colored region indicate the $90 \%$ confidence region for the fits to the submillimeter SED. In panel a)-c) the effective temperature of the central blackbody is $5000 \mathrm{~K}$, in d) it is taken to $100 \mathrm{~K}$. The best fit to the combined submillimeter and mid-infrared datasets is shown in panel c) and d) with the white "+". In c) the model of Schöier et al. (2004) fitting the submillimeter SED and interferometer data is shown with the black "+". 


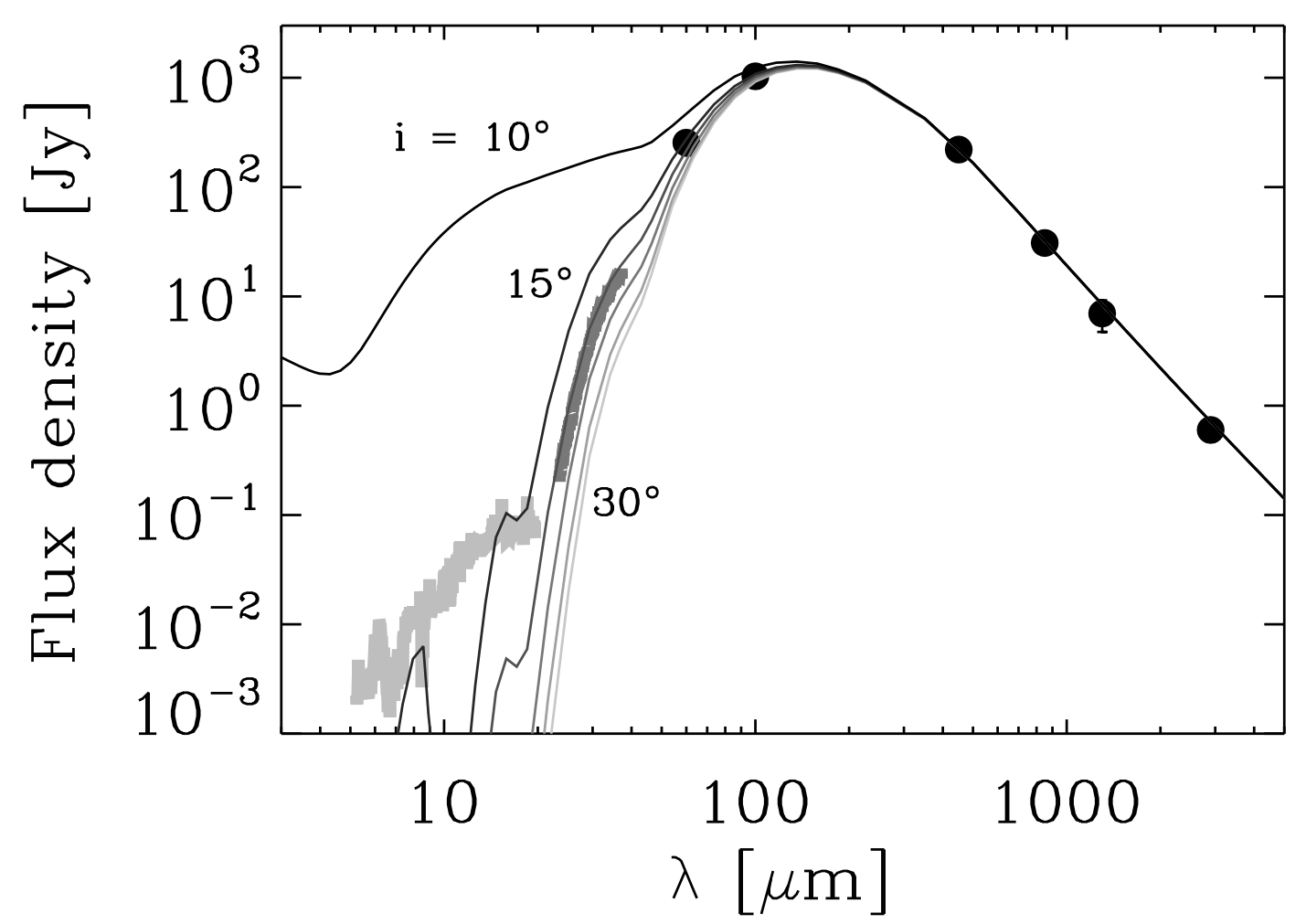

Fig. 3.- Envelope models with $R_{\mathrm{i}}=32 \mathrm{AU}$ and a conical outflow cavity with an opening angle of $30^{\circ}$ and inclination angles of $10^{\circ}, 15^{\circ}, 17^{\circ}, 20^{\circ}, 25^{\circ}$ and $30^{\circ}$ (going from dark to light grey). The IRS detection and upper limits are shown by the dark and light grey lines as in Fig. 1. 


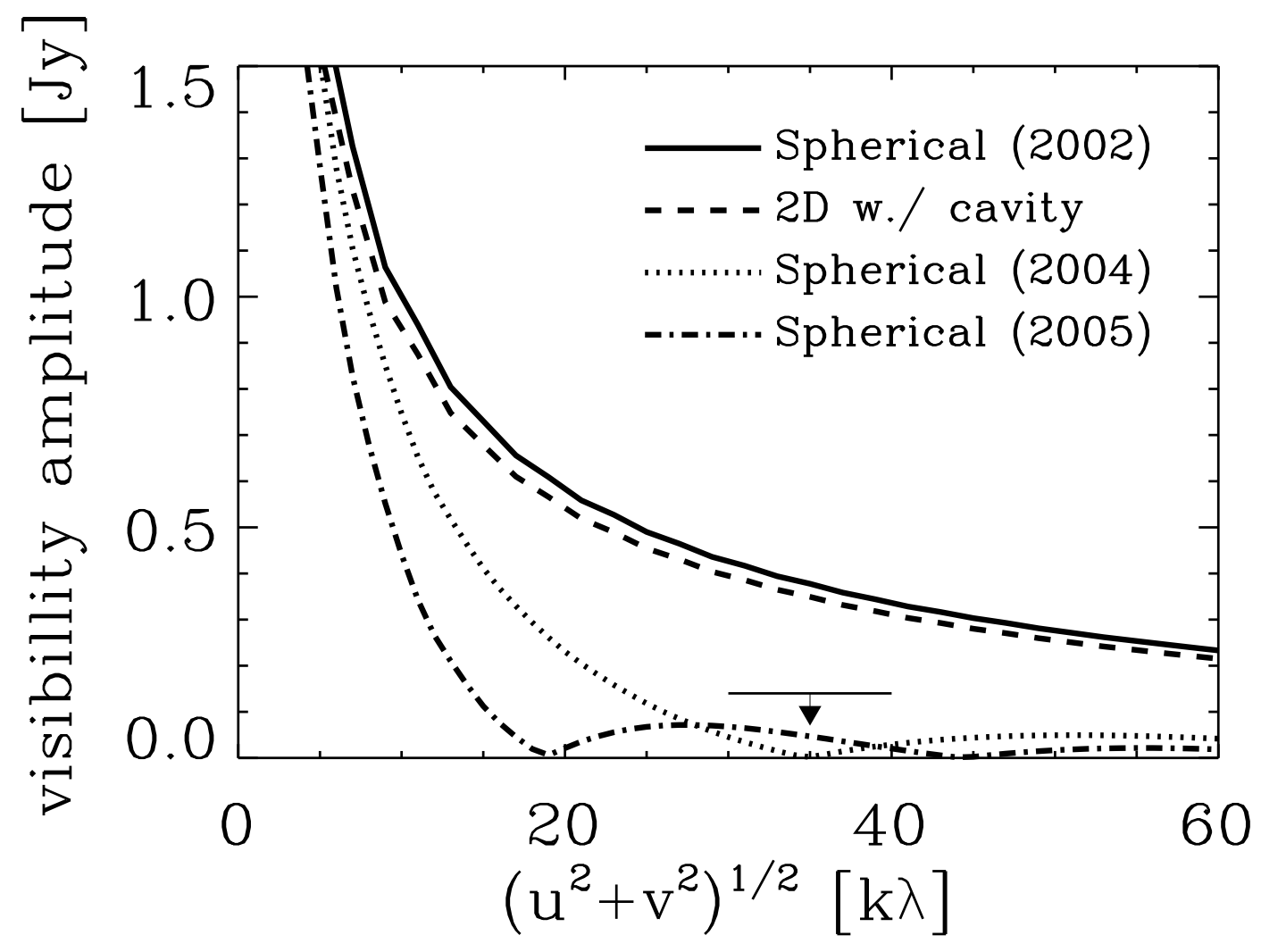

Fig. 4.- Visibility amplitudes versus projected baseline lengths at $1.37 \mathrm{~mm}$. The phase center is taken to be the center point of the circumbinary envelope, i.e., between the two binary components (see Schöier et al. 2004). Shown are the 1D spherical model of Schöier et al. (2002) (solid line), the models of Schöier et al. (2004) and this paper with larger inner cavities (dotted and dashed-dotted, respectively) and the 2D outflow cavity model (dashed). Note that the outflow cavity and spherical models with $R_{\mathrm{i}}=32 \mathrm{AU}$ are almost indistinguishable and overproduce the emission at the $30-40 \mathrm{k} \lambda$ baselines compared to the OVRO observations of Schöier et al. (2004) (indicated by the horizontal line and downward arrow). 